\title{
Development of a computationally efficient bubble column simulation approach by way of statistical bubble micro-flow modelling
}

\author{
W. Coetzee ${ }^{1}$, R. L. J. Coetzer ${ }^{2}$ \& R. Rawatlal ${ }^{1}$ \\ ${ }^{I}$ Department of Chemical Engineering, University of Cape Town, \\ South Africa \\ ${ }^{2}$ Sasol Technology Research and Development, South Africa
}

\begin{abstract}
Bubble columns are extensively used in the chemical process industry, however their hydrodynamics are computationally expensive to simulate, severely restricting design and optimisation studies. A novel discrete-phase bubble column modelling approach is proposed where the flow-field in the immediate vicinity of individual bubbles is predicted from the Reynolds number via an algebraic bubble flow model, i.e. the Bubble Cell Model (BCM). This contrasts to the traditional Euler-Lagrange approach, where the flow structure is resolved at a larger scale using the Navier-Stokes equations. The construction of the BCM involves generating the micro-flow fields through solving the analogous case of the flow over a fixed sphere with the Navier-Stokes equations at 22 equally spaced Reynolds numbers in the operating range of $R e \leq 270$. The model construction then occurs in two stages, i.e. stage one: the fitting of each velocity vector field at the discrete Reynolds numbers, and stage two: the cross correlation of the stage one model parameters with respect to Reynolds number. For stage one, a hybrid between analytical and statistical models was found to provide accurate fits, resulting in $R^{2}$ values ranging from 0.9968 to 0.9999 . The Reynolds dependence of the parameters was found to be described through simple polynomial and exponential models in the second stage construction, producing a model which generates the velocity vector field around a bubble for a given Reynolds number as the result of a single algebraic evaluation. The integration of the BCM with a Eularian macro fluid model has also been investigated for a single bubble pilot test case.
\end{abstract}

Keywords: bubble flow, Euler-Lagrange, cell model. 


\section{Introduction}

Bubble column simulation requires the resolution of complex hydrodynamic interactions between the gas and liquid phases. This can be modelled with the Navier-Stokes equations, however, the solving of the Navier-Stokes equations at bubble scale, e.g. Volume-Of-Fluid method, is impractical with regards to computational expense when considering any more than a few bubbles [1]. The momentum source generated by the bubbles on the continuum fluid phase is subsequently modelled through experimentally correlated force models i.e. drag, lift and added mass, which is used in coupling the gas phase to the continuum [2]. The gas phase can then either be tracked as individual bubbles, i.e. the EulerLagrange approach, or approximated as a continuum i.e. the Euler-Euler approach. These approaches enable larger grid sizes and make the simulation of entire bubble columns possible. However, the bubble scale flow detail is severely reduced and computational efficiency remains a problem [3]. Furthermore, the question of which physical effects to include in the coupling model, is still under debate [4].

It follows that the choice of bubble column simulation approach depends on the level of flow detail required and the size of the geometry, as illustrated in Figure 1. The computational expense required for large sized problems can subsequently inhibit the choice of smaller scale models.

In an attempt to address these issues, relating to multiphase flow simulation in general, it has been proposed by the authors to substitute the flow-fields in the direct vicinity of the bubbles in a Euler-Lagrangian framework, with an algebraic micro-flow model correlated to the Navier-Stokes solution for the appropriate flow situations. The algebraic flow model will be referred to as the Bubble Cell Model (BCM). The computationally expensive regions around individual bubbles are therefore accounted with the BCM, which can be rapidly evaluated, in contrast to the computationally expensive partial differential Navier-Stokes equations. In addition, the $\mathrm{BCM}$ can be used to determine the force acting on the bubble

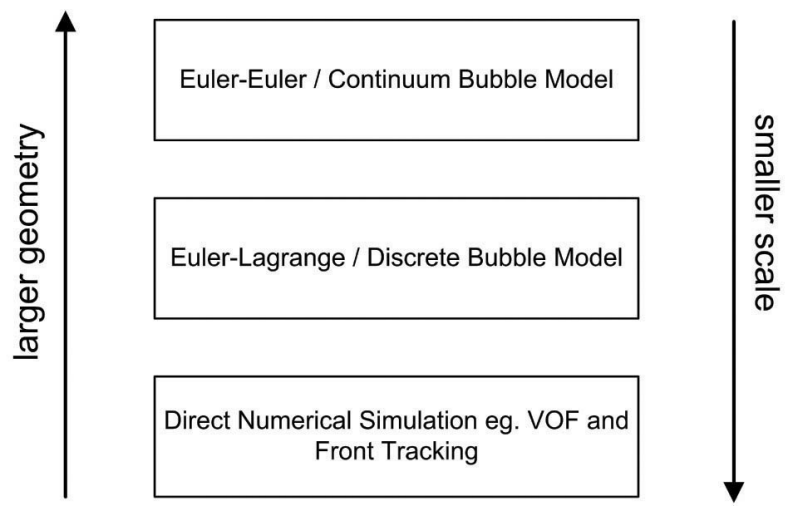

Figure 1: CFD bubble column simulation framework. 
in the equation of motion and allows the bubble scale flow information to be approximated.

\section{Bubble cell model development}

The aim of the BCM is to provide a model of the velocity vector field, $\mathbf{u}^{\mathrm{M}}$, with respect to spatial position, $\mathbf{x}$, around a bubble as a function of Reynolds number, $R e=\frac{\rho D u_{r e l}}{\mu}$ (where $\rho, D, u_{r e l}$ and $\mu$ represent the fluid density, bubble diameter, bubble relative velocity and fluid viscosity respectively). The solution to the Navier-Stokes equations for the flow over a fixed sphere with appropriate boundary conditions (as discussed in Section 2.1), is regarded as the "true velocity field", $\mathbf{u}^{\text {true }}$, with respect to the construction of the BCM. To approximate $\mathbf{u}^{\text {true }}$ as a function of Reynolds number, a two stage fitting strategy has been devised. Stage one involves fitting the velocity vector fields at discrete Reynolds numbers, whilst stage two aims to cross correlate the parameters of the stage one model w.r.t. Reynolds number. This strategy is schematically presented in Figure 2.

\subsection{Flow over fixed sphere: data generation}

To set up the case, the boundary condition at the bubble surface and the bubble shape is considered:

- If the liquid is pure enough, it is possible for it to slip along the surface of the bubbles (free slip condition), in contrast to the flow past rigid bodies, where slip will not occur (no-slip condition). This very often makes the flow unseparated in circumstances where the flow around a solid body of similar shape would be separated (i.e. the fluid flow becomes detached from the surface of the object at higher Reynolds numbers) or even turbulent [5]. Consequently, the boundary condition for a contaminant free system,

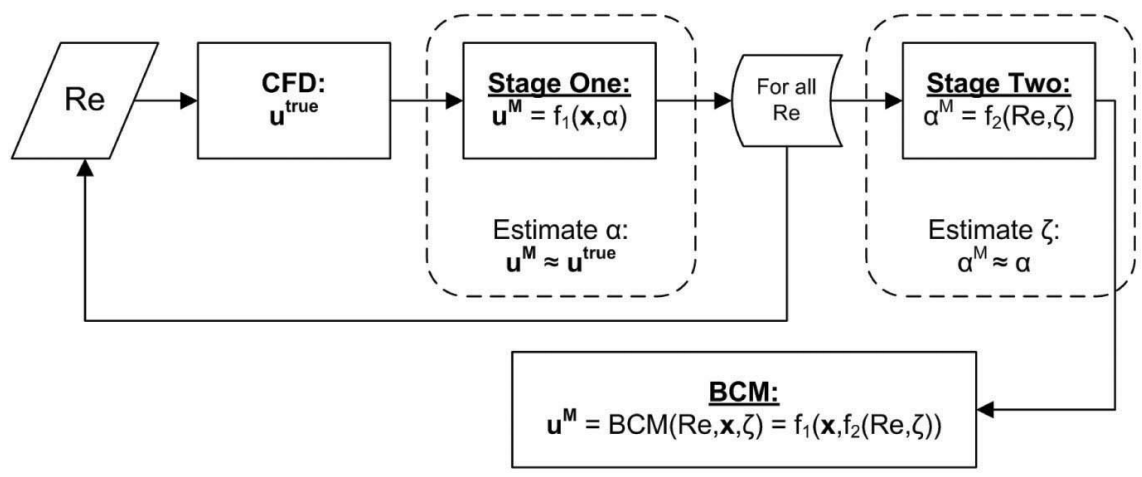

Figure 2: BCM two stage fitting strategy, where $f_{1}, \alpha, f_{2}$ and $\zeta$ represent the overall stage one model, stage one parameters, stage two model and stage two parameters respectively. 
imposed at the bubble surface on the tangential component of the liquid velocity, is a zero-shear-stress one rather than a no-slip one [6].

- The shape of the bubble directly influences the local hydrodynamics and can be transient during bubble motion. According to the research of Tomiyama et al. [7], bubble motion, shape and velocity are remarkably sensitive to initial shape deformation. The researchers subsequently proposed a steady state drag model which is dependent on bubble shape through the dimensionless Eötvös number $\left(E \ddot{o}=\frac{\Delta \rho g D^{2}}{\sigma}\right.$, with $\Delta \rho, g$ and $\sigma$ representing the density difference, gravity vector and surface tension respectively). It has become apparent that bubble shape is an important parameter which adds new degrees of freedom to an already complex problem.

For the development of the BCM approach, we restrict the focus onto spherical bubbles in a contaminant free system. It follows from experimental data [8], that the spherical assumption is valid for $R e \leq 270$ for an air-water system, whilst the clean system will be accounted for with an zero-shear-stress boundary condition on the bubble surface. Furthermore, to allow for faster development of the concept, the work will be carried out in two dimensions. It should be noted that the approach could be extended to different shaped bubbles occurring in heterogeneous bubble columns through parametrization of the Eö number, higher Reynolds numbers and three dimensions.

The Navier-Stokes equations are solved with the Finite-Volume method, using the SIMPLE pressure-velocity coupling scheme [9] and 2nd order UPWIND spatial discretization in the Fluent 12 solver (FLUENT INC, USA). The mesh is created according to the geometry depicted in Figure 3, and its resolution optimized according to the convergence of the drag force on the bubble surface.
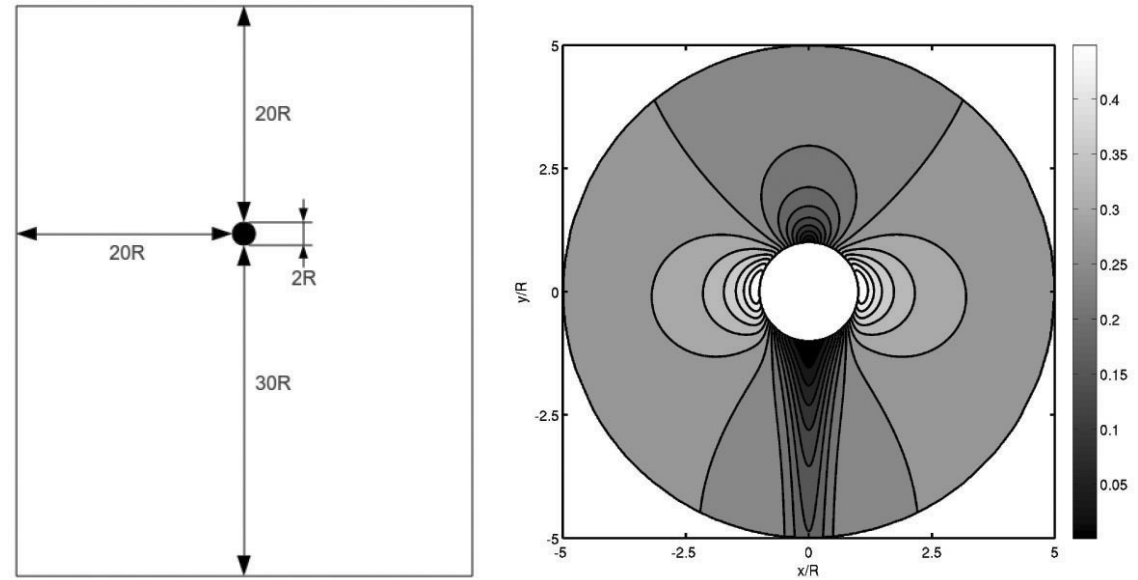

Figure 3: Case geometry and resulting velocity magnitude contours following CFD simulation. The geometry is set up with respect to the radius (R) of the bubble and the ratios chosen to allow flow dynamics to sufficiently develop across the domain. 
This resulted in 164,983 data points in the BCM region, which was chosen as 5 times the bubble radius. The drag coefficients for the Reynolds numbers evaluated, corresponded to the experimental data for bubbles in pure systems [10].

\subsection{Stage one model}

\subsubsection{Analytical solutions}

Analytical solutions provide a good starting point to the development of the BCM, since they capture useful features of the flow structure. Solutions are possible through simplifying assumptions, such as either creeping flow (i.e. zero advective terms), or potential flow (i.e. zero viscous terms), making analytical evaluation of the steady state, incompressible Navier-Stokes equations (eqn. (1) and (2)) possible. Here $\mathbf{u}, p$ and $\mathbf{S}$ refer to the fluid velocity vector, fluid pressure and the body force vector respectively.

$$
\begin{gathered}
\nabla \cdot \mathbf{u}=0 \\
\underbrace{\rho(\mathbf{u} \cdot \nabla \mathbf{u})}_{\text {advective terms }}=-\nabla p+\underbrace{\mu \nabla^{2} \mathbf{u}}_{\text {viscous terms }}+\underbrace{\mathbf{S}}_{\text {body forces }}
\end{gathered}
$$

The creeping flow solution (valid at $R e \ll 1$ ) was derived by Stokes [11], by assuming axisymmetric flow and writing the Navier-Stokes equations in terms of the Stokes stream function $\psi$ (the two velocity components are expressed as derivatives of $\psi$ such that continuity is automatically satisfied) in spherical coordinates ( where $\mathbf{r}$ and $\boldsymbol{\theta}$ correspond to the radial and angular spatial components, centred around the centre of volume of the bubble), simplifying the problem considerably. Following the solution strategy by Slattery [12], the stream function is proposed as a fourth order polynomial whose coefficients are obtained through the substitution of the boundary conditions. Applying the zero-shear-stress boundary conditions, eqn. (3)-(5), the stream function is obtained, which upon differentiation with respect to $\boldsymbol{\theta}$ and $\mathbf{r}$ leads to radial and angular velocities, $\mathbf{u}_{\mathbf{r}}$ and $\mathbf{u}_{\theta}$, which are subscripted for the creeping flow solution as $\mathbf{u}_{\mathbf{r}, \mathbf{C F}}$ and $\mathbf{u}_{\theta, \mathbf{C F}}$ in eqn. (6) and (7). The free stream velocity here, $u_{\infty}$, corresponds to the relative bubble velocity $u_{\text {rel }}$.

$$
\begin{gathered}
\mathbf{u}_{\left.\mathbf{r}\right|_{\mathbf{r} \rightarrow \infty}}=u_{\infty} \cos (\theta) \\
\mathbf{u}_{\left.\mathbf{r}\right|_{\mathbf{r}=\mathbf{R}}}=0 \\
\frac{d \mathbf{u}_{\left.\theta\right|_{\mathbf{r}=\mathbf{R}}}}{d \mathbf{r}}=0 \\
\mathbf{u}_{\mathbf{r}, \mathbf{C F}}(r, \theta)=u_{\infty}\left[1-\frac{3}{4}\left(\frac{R}{\mathbf{r}}\right)-\frac{1}{4}\left(\frac{R}{\mathbf{r}}\right)^{3}\right] \cos (\boldsymbol{\theta}) \\
\mathbf{u}_{\theta, \mathbf{C F}}(r, \theta)=u_{\infty}\left[1-\frac{3}{8}\left(\frac{R}{\mathbf{r}}\right)+\frac{1}{8}\left(\frac{R}{\mathbf{r}}\right)^{3}\right] \sin (\boldsymbol{\theta})
\end{gathered}
$$


Although zero viscosity fluids do not actually exist, the assumption (valid at $R e \rightarrow \infty$ ) provides a reasonably good description of the velocity profile, except near the body and beyond the line of flow separation [13]. The potential flow solution is derived by defining velocity potential $\Phi$, such that $\mathbf{u}=-\nabla \Phi$, the zero-shear-stress boundary condition, is automatically satisfied by the inviscid assumption. For incompressible potential flows, $\Phi$ satisfies Laplace's equation which can be solved with the method of separation of variables [12], and upon differentiation yields the potential flow velocity fields, $\mathbf{u}_{\mathbf{r}, \mathbf{P F}}$ and $\mathbf{u}_{\theta, \mathbf{P F}}$, as presented in eqn. (8) and (9).

$$
\begin{aligned}
& \mathbf{u}_{\mathbf{r}, \mathbf{P F}}(r, \theta)=u_{\infty}\left[-1+\left(\frac{R}{\mathbf{r}}\right)^{3}\right] \cos (\boldsymbol{\theta}) \\
& \mathbf{u}_{\theta, \mathbf{P F}}(r, \theta)=u_{\infty}\left[1+\frac{1}{2}\left(\frac{R}{\mathbf{r}}\right)^{3}\right] \sin (\boldsymbol{\theta})
\end{aligned}
$$

The solutions for both simplifying assumptions are simple algebraic expressions which allow for rapid numerical function evaluation. However, neither of these solutions are able to capture the flow separation and wake features which are artefacts arising when $R e>1$, resulting in poor flow prediction at the rear of the bubble and incorrect hydrodynamic force calculation which limits the practical applicability of these solutions.

\subsubsection{Statistical modelling}

The need for statistical modelling arises from an attempt to bridge the gap between the two extrema cases of the analytical solutions i.e. $R e=0$ and $R e=\infty$, by accounting for features occurring in the non-idealized flow regimes. Since the aim of the BCM is rapid functional evaluation, a semi-analytical model, expressed through algebraic expressions, would be justified.

The two analytical models, associated with creeping and potential flow, is to be combined to take account of a significant portion of the flow structure. A linear combination of the two solutions is used, i.e. eqn. (10) and (11), where $\alpha_{1,1}$ to $\alpha_{2,2}$ represents the weighting coefficients obtained through linear least squares with respect to $\mathbf{u}^{\text {true }}$. For this purpose, only data from the region $\pi / 2 \leq \theta \leq 3 \pi / 2$ is considered, since this region is best represented by the two solutions, whereas the wake feature at the rear of the bubble negatively impacts the least squares fit by attempting to take account of a structure the model is not capable of representing.

$$
\begin{aligned}
& \mathbf{u}_{\mathbf{r}, \mathbf{A}}^{\mathbf{M}}=\alpha_{1,1} \mathbf{u}_{\mathbf{r}, \mathbf{C F}}+\alpha_{1,2} \mathbf{u}_{\mathbf{r}, \mathbf{P F}} \\
& \mathbf{u}_{\theta, \mathbf{A}}^{\mathbf{M}}=\alpha_{2,1} \mathbf{u}_{\theta, \mathbf{C F}}+\alpha_{2,2} \mathbf{u}_{\theta, \mathbf{P F}}
\end{aligned}
$$

The residuals to these solutions, i.e. eqn. (12) and (13), require statistical modelling to be accounted for. This has been achieved by considering each of the residual velocity surfaces, $\mathbf{u}_{\mathbf{r}, \mathbf{r e s}}$ and $\mathbf{u}_{\theta}$,res across the Reynolds operating range, and identifying regions following simple functional forms. In addition, the 

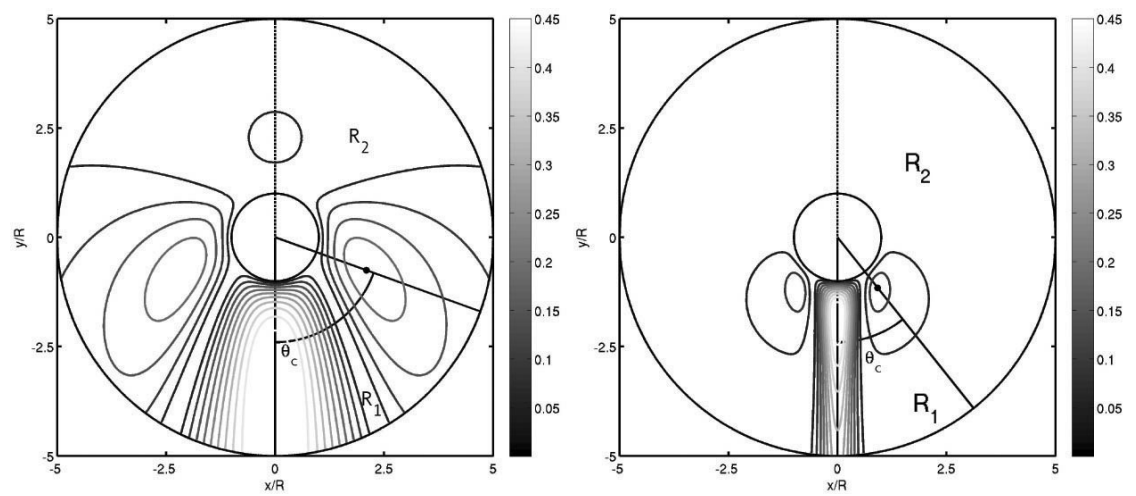

Figure 4: Contours of $\left|\mathbf{u}_{\mathbf{r}, \text { res }}\right| / u_{\infty}$, illustrating the change of $\theta_{c}$ w.r.t. Reynolds number. $R_{1}$ and $R_{2}$ denote region 1 and region 2 .
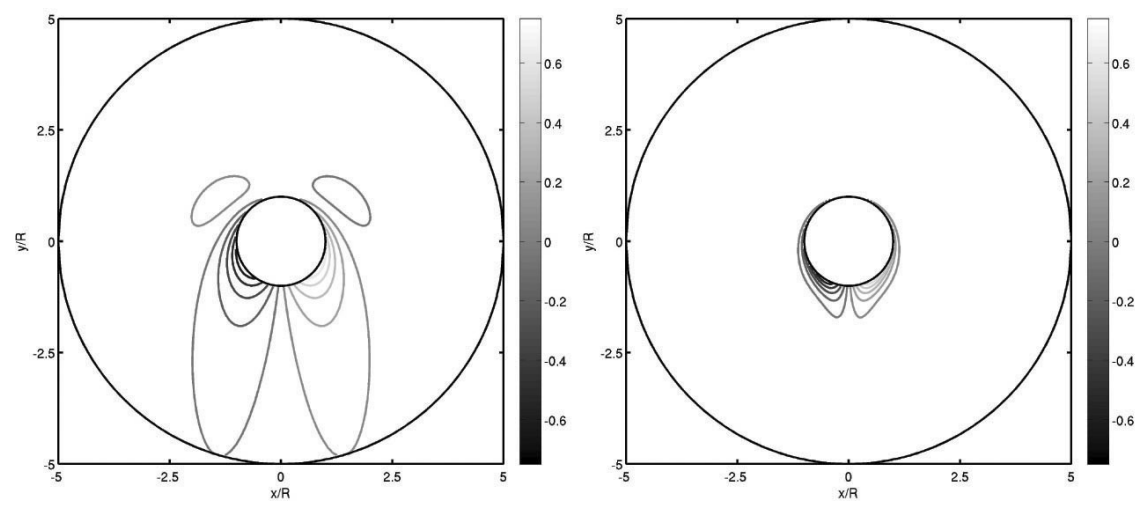

Figure 5: Contours of $\mathbf{u}_{\theta, \text { res }} / u_{\infty}$ at two different Reynolds numbers.

symmetry across the $x=0$ line, was also used to simplify the problem.

$$
\begin{aligned}
& \mathbf{u}_{\mathbf{r}, \text { res }}=\mathbf{u}_{\mathbf{r}}^{\text {true }}-\mathbf{u}_{\mathbf{r}, \mathbf{A}}^{\mathrm{M}} \\
& \mathbf{u}_{\theta, \text { res }}=\mathbf{u}_{\theta}^{\text {true }}-\mathbf{u}_{\theta, \mathbf{A}}^{\mathrm{M}}
\end{aligned}
$$

The residual of to the radial velocity, was largely focussed in the bubble wake region. This was captured by identifying a critical angle, $\theta_{c}$, corresponding to the maximum of the local peak at the rear of the bubble, which divides the domain into two regions, as illustrated in Figure 4. These regions were subsequently captured through a series of exponential models. The final model for $\mathbf{u}_{\mathbf{r}}^{\mathbf{M}}$ had a total of 17 parameters $\left(\alpha_{1, i}\right)$ and resulted in $R^{2}$ values between $0.9968-0.9999$. The residual of the angular velocity was a maximum at the bubble surface, which decayed into the radial direction. This surface was approximated through finding the appropriate model to represent $\left.\mathbf{u}_{\theta, \text { res }}\right|_{\mathbf{r}=\mathbf{R}}$ and fitting the exponential decay of this model 
Table 1: Surface fit results of $\mathbf{u}_{\mathbf{r}}^{\mathbf{M}}$ and $\mathbf{u}_{\theta}^{\mathbf{M}}$ with varying Reynolds number.

\begin{tabular}{lllll}
\hline $\mathrm{Re}$ & $\mathbf{u}_{\mathbf{r}}^{\mathrm{M}}: \mathrm{MSE}$ & $\mathbf{u}_{\mathbf{r}}^{\mathrm{M}}: R^{2}$ & $\mathbf{u}_{\theta}^{\mathrm{M}}: \mathrm{MSE}:$ & $\mathbf{u}_{\theta}^{\mathrm{M}}: R^{2}$ \\
\hline 0.1 & $3.1015 \mathrm{e}-12$ & 0.9968 & $8.1015 \mathrm{e}-12$ & 0.9974 \\
15 & $7.2915 \mathrm{e}-08$ & 0.9989 & $2.9301 \mathrm{e}-07$ & 0.9978 \\
75 & $3.9737 \mathrm{e}-07$ & 0.9998 & $1.7457 \mathrm{e}-06$ & 0.9995 \\
135 & $7.0539 \mathrm{e}-07$ & 0.9999 & $3.9877 \mathrm{e}-06$ & 0.9997 \\
195 & $1.2357 \mathrm{e}-06$ & 0.9999 & $6.9260 \mathrm{e}-06$ & 0.9997 \\
270 & $2.2273 \mathrm{e}-06$ & 0.9999 & $1.1348 \mathrm{e}-05$ & 0.9998 \\
\hline
\end{tabular}

radially. This resulted in a reasonable approximation of the data with $R^{2}=0.9974$ to 0.9998 , with 11 parameters $\left(\alpha_{2, i}\right)$. (Please note that due to space restrictions it is not possible to expand on the residual models i.e. $\mathbf{u}_{\mathbf{r}, \text { res }}^{\mathrm{M}}$ and $\mathbf{u}_{\theta, \text { res }}^{\mathrm{M}}$. They will however be discussed in more detail in an upcoming paper by the authors.)

The overall results are presented in Table 1. It is seen that the accuracy of the approximation increases with Reynolds number. This is due to the deviations from the potential flow solution, mainly consisting of the wake feature, becoming concentrated in a smaller region as $R e$ increases, thereby allowing for a more accurate fit. At the lower range the wake is more spatially spread out, and whilst the $\mathbf{u}_{\mathbf{r}, \mathbf{A}}^{\mathrm{M}}$ and $\mathbf{u}_{\theta, \mathbf{A}}^{\mathrm{M}}$ approximations becomes better as the creeping flow solution is approached at $R e=0.1$, the $\mathbf{u}_{\mathbf{r}, \text { res }}^{\mathrm{M}}$ and $\mathbf{u}_{\theta, \text { res }}^{\mathrm{M}}$ models are not as accurate at lower Reynolds numbers. Response surface strategies have also been evaluated for the same data, however they resulted in higher MSE's with more parameters [14].

\subsection{Stage two model}

To ensure a smooth response of the first stage parameters, $\alpha_{i, j}$, with respect to Reynolds number, each first stage fit was carried out using the parameter estimations from the previous fit as initial guesses, thereby warm starting the optimization e.g. the parameter estimates for the $R e=270$ surface is used as initial guesses for the $R e=255$ surface. Carrying out the procedure from high to low Reynolds numbers provided the most well behaved curves. The dependence of the $\alpha^{\prime} s$ w.r.t. $R e$ was found to be described by first and second order polynomial and exponential models. A typical example of the behaviour of the $\alpha^{\prime} s$ is given in Figure 6.

The final parameters to the BCM was found by optimizing all of the $\zeta$ parameters with respect to the original CFD surfaces i.e. $\mathbf{u}^{\text {true }}$. This results in a bubble flow field model dependent on the Reynolds number ( $R e$ ), spatial position $(r$ and $\theta$ ) and the stage two fitting parameters $(\zeta)$. 

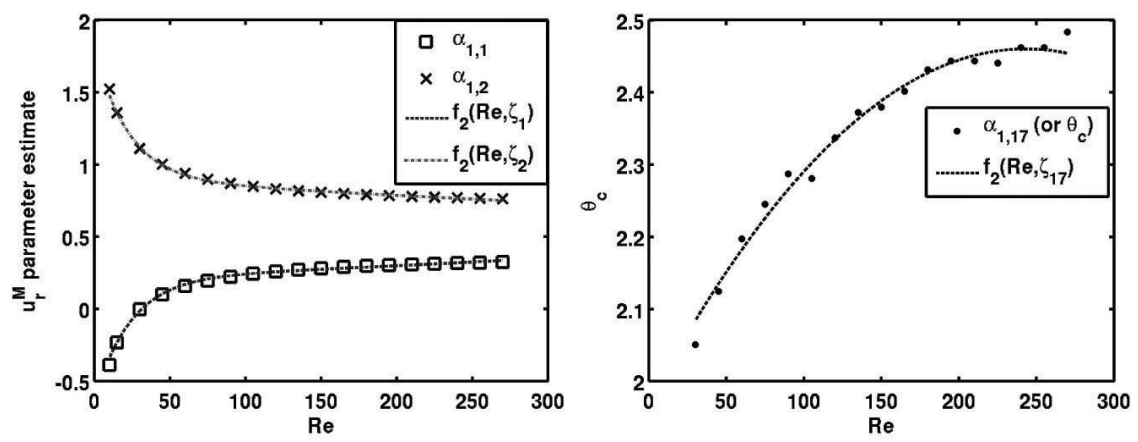

Figure 6: Second stage fit of parameters $\alpha_{1,1}, \alpha_{1,2}$ and $\alpha_{1,17}$.

\section{Integration of bubble cell model with macro flow model: single bubble test case}

With the BCM constructed, the next step is to integrate it with a macro flow model to account for the continuum outside of the BCM regions. For the development of this model, the Openfoam C++ libraries where used [15]. The BCM regions are tracked in a Lagrangian framework, and the Navier-Stokes equations solved on a structured grid for the continuum. A structured grid is used such that the computational cells falling within the BCM regions can be identified, through their structured relationship, in a computationally efficient manner. To exclude the BCM regions from the Eularian flow computations, the linear system of equations is modified. The corresponding values are set to that of the BCM and the cells are eliminated from the equations. Furthermore, the edges of the $\mathrm{BCM}$ regions neighbouring the computational cells, act as "internal boundary conditions", thereby closing the problem.

As a pilot test to the BCM approach, the case of a single bubble rising is considered with a fixed Reynolds number as illustrated in Figure 7. The resulting flow field in the continuum qualitatively follows experimental and numerical modelling trends $[16,17]$. A rigorous analysis and validation of the results however, remain to be completed at this stage. Furthermore, the equation of motion for the BCM case needs to be developed.

\section{Conclusion}

A novel bubble column modelling approach is proposed, with the goal to offer bubble scale flow information at reduced computational expense. This is aimed to be achieved through the introduction of an algebraic flow model which approximates the flow field around individual bubbles, i.e. the BCM, in contrast to having to solve a set of non-linear PDE's. The BCM was constructed through a two stage fitting process, where the parameters are correlated with CFD generated 


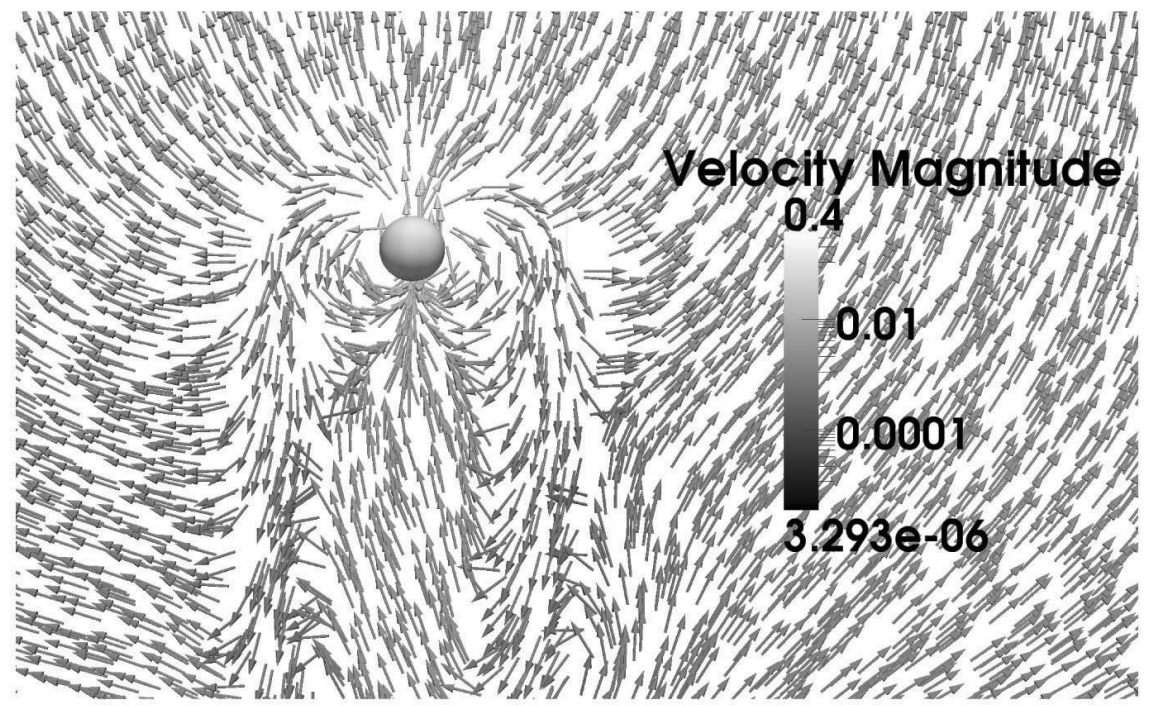

Figure 7: Resulting velocity vector field of a single bubble rising in a EulerLagrange framework following the BCM approach. The bubble is simulated as $R e=120$.

data, for the analogues situation of flow over a fixed fluid sphere. The combination of the creeping and potential flow analytical solutions provided a good starting point by taking account of a large portion of the flow field. The non-linear deviations occurring outside of their idealized operating regimes however, required the introduction of statistical models. The combination of these models resulted in a semi-analytical model capable of approximating the flow field very accurately in the range examined. Furthermore, the cross correlation of the stage one parameters with Reynolds number proved to be successful with simple polynomial and exponential functions, thereby arriving at the BCM.

The BCM has been integrated with a "macro Eularian" flow model, whereby the $\mathrm{BCM}$ regions are tracked in a Lagrangian framework. This has been performed successfully with a pilot test case, where a single bubble rising is simulated with a fixed Reynolds number. Rigorous validation remains outstanding, however, the results qualitatively appear realistic and illustrates the potential of the approach.

\section{References}

[1] Brenner, C.E., Fundamentals of Multiphase Flow. Cambridge University Press, 2005.

[2] Michaelides, E.E., Particles, Bubbles \& Drops: Their Motion, Heat and Mass Transfer. World Scientific, 2006.

[3] Joshi, J.B., Computational flow modelling and design of bubble column reactors. Chemical Engineering Science, 56, pp. 5893-5933, 2001. 
[4] Sokolichin, A., Eigenberger, G. \& Lapin, A., Simulation of buoyancy driven bubbly flow: Established simplifications and open questions. American Institute of Chemical Engineers, 50, pp. 24-45, 2004.

[5] Magnaudet, J. \& Eames, I., The motion of high-Reynolds-number bubbles in inhomogeneous flows. Annual Review: Fluid Mechanics, 32, pp. 659-708, 2000.

[6] Batchelor, G.K., An Introduction to Fluid Dynamics. Cambridge University Press, 1967.

[7] Tomiyama, A., Celata, G.P., Hosokawa, S. \& Yoshida, S., Terminal velocity of single bubbles in surface tension force dominant regime. International Journal of Multiphase Flow, 28, pp. 1497-1519, 2002.

[8] Clift, R., Grace, J.R. \& Weber, M.E., Bubbles, Drops and Particles. Academic Press, 1978.

[9] Pantankar, S.V., Numerical Heat Transfer and Fluid Flow. Taylor \& Francis, 1980.

[10] Haberman, W.L. \& Morton, R.K. David Taylor Model Basin Rep, 802, 1953.

[11] Stokes, G.G., On the effect of the internal friction of fluids on the motion of a pendulum. Transactions of the Cambridge Philosophical Society, 9, pp. $8-106,1851$.

[12] Slattery, J.C., Advanced Transport Phenomena. Cambridge University Press, 1999.

[13] Bird, R.B., Steward, W.E. \& Lightfoot, E.N., Transport Phenomena: second edition. John Wiley \& Sons Inc, 2002.

[14] Coetzee, W., Coetzer, R.J.L. \& Rawatlal, R., An analysis of response surface strategies in developing statistical models for application in bubble column modelling. submitted to: Computers \& Chemical Engineering, 2010.

[15] OpenCFD, http://www.openfoam.com/,OpenFoam - The Open Source CFD Toolbox, 2009.

[16] Ellingsen, K. \& Risso, F., On the rise of an ellipsoidal bubble in water: oscillatory paths and liquid-induced velocity. Journal of Fluid Mechanics, 440, pp. 335-268, 2001.

[17] Deen, N.G., van Sint Annaland, M. \& Kuipers, J.A.M., Multi-scale modeling of dispersed gas-liquid two-phase flow. Chemical Engineering Science, 59, pp. 1853-1861, 2004. 